\title{
Magnar Brekke, Øivin Andersen, Trine Dahl and Johan Myking: Applications and Implications of Current LSP Research. Bergen: University of Bergen, 1994
}

These two volumes are the proceedings of the 9th European Symposium on Languages for Special Purposes held in Bergen in 1993. The editors chose not to issue a 'slim volume' of selected highlights, but to publish most of the papers to give a broad overview of the various fields of LSP. The result is two thick volumes presenting more than a hundred papers.

Hence not all of the papers can be discussed. In fact the book's status as non-selective conference proceedings means that the volumes contain a number of immature articles. Reading the volumes through is like attending a conference where it is mercifully possible to escape when one has chosen the wrong session. Most papers are in English, a few in German, and one or two in French and Spanish. While there are a few misprints, one article has all its tables omitted, and there are occasional patches of bad English, the editors have fundamentally done a wonderful job in getting all this material readable, clearly printed, and firmly bound (all 969 pages!)

A broad overview should give one a sense of the nature of the field, and many of the papers do give introductions or surveys of subfields, so that a review allows one to consider what LSP studies were in 1993.

The collection covers both 'applications' and 'implications' of LSP studies; it recognises the origins of LSP studies in an up-valuation of the 'useful arts' in relation to the 'liberal' (Kalverkämper 1992), and covers a wide range from the loftily 'liberal' implications to the 'useful' applications. However even the 'pure' work is a mixed bag characterised by the object of study rather than the method. This object is communication in 'specialist' domains, where 'specialist' has something to do with having a body of knowledge which is not 'general knowledge'. Within this remit, it concentrates on (Western) languages and domains which have economic influence or importance. While anthropological linguistics looks out from well-known and described languages on more obscure and marginal ones, in LSP studies as represented here speakers of some fifteen well-known languages look in on language practices in a few of the best-described (predominantly English and German in this North European environment, also French and Russian, with some Spanish, Italian, Swedish, and a few others).

The traditional interests of academics predominated in the conference and perhaps still do in academic LSP studies. So, although there are several papers on business and legal communication and several on spoken genres and registers, the domain most frequently discussed is science/technology and the 
medium is writing. To judge from these papers the applications of European LSP are in foreign language teaching, translation, terminology, lexicography, and its implications in studies of ideology and the history of ideas. Only two papers deal with technical writing and none with mother-tongue communication training or industrial language - language training of immigrants, etc., for the workplace; there are connections to be made here that would probably be valuable for both sides.

The volume is introduced by a series of plenary papers. A good one to start with is that by Laurén, which traces the development of LSP traditions since the nineteenth century: Wörter und Sachen studies in Scandinavian dialectology and in Wirtschaftslinguistik as the ancestors of linguistically-oriented studies of contrastive culture and of professional practices; philosophical aspects, and Wüster's formulation, of terminology; the history of learning the parallel development of vernacular technolects and science in the Renaissance, for example; similarly Fachprosaforschung, investigating the academic literature of the Middle Ages; the Prague structuralists' interest in the structuring and adaptation of language to particular (intellectual) functions; the shift from concern with specialist language to concern with specialist texts; and the corresponding application of computer-based corpora in the investigation of parole. Laurén sees teaching method as one area of application of these theoretical studies but scarcely mentions others; he is mainly concerned with the cultural and philosophical implications of LSP studies viewed as an intellectual endeavour reaching back to Aristotle, carried on mainly in Central and Eastern Europe, and by no means always putting itself at the disposal of economic gain.

A paper emphasising diversity like Laurén's raise the question of what sort of discipline LSP is. Citkina's plenary unconvincingly places it in the early stages of Kuhn-style schemata for the development of sciences but usefully identifies interlingual comparison as a key activity and stresses the 'application-oriented' nature of much LSP work - the 'pure' researcher usually has a use in mind. Her principles for comparative LSP research underline the importance of inclusiveness and making use of all the investigatory tools linguistics (and discourse analysis, etc.) make available.

The issue of the nature of LSP is illuminated by some historical papers on linguistic aspects of discipline formation. Gunnarson and her group organised a workshop on their impressive project on the development of academic Swedish at a variety of cognitive-linguistic levels. Gunnarson's own article deals qualitatively with the development of economics writing from an eighteenthcentury 'pre-stage' through an 'establishing stage' around 1900 to its present 'specialised stage'. The progression is marked by, among other things, increasing reference to other specialists' work, and to discipline-internal concepts, 
accompanied by decreasing reference to the real world (as a source of analogies for example) and the elimination of 'preaching'. The interesting point is that medical and technical articles were already in the 'establishing' stage when economics was still in the 'pre-stage'.

This view, is however, in need of balance from an alternative, non-teleological, perspective. What Gunnarson calls 'specialisation' (and Citkina's teleology) needs interpretation in terms of Becher's four dimensions (Becher 1989): 'pure-applied' (physics vs. engineering); 'hard-soft' (cumulative knowledge like physics vs. changing perspectives like literary studies); convergentdivergent (convergent subjects have single models and aims and tend towards orthodoxy, divergent ones have multiple models and aims and resemble loose federations, so physics is convergent and geography divergent), and 'urbanrural' (in urban subjects the number of research topics is small relative to the number of researchers, in 'rural' it is large, so physics is urban and biology is rural). Her linguistic findings from economics represent its 'urbanisation' and development towards greater 'purity' and 'hardness' rather than progress towards a necessary goal; the absence of many of these markers from literary studies represents divergency and rurality rather than lack of specialisation or a primitive evolutionary position.

Analysing LSP studies in Becher's terms, we have to separate 'pure' investigations from their application. and assign the applications to other disciplines. Even though the same people are doing the research and applying it, they are using linguistic (etc.) methods and principles in research and quite different ones in application. The remaining 'pure' field is highly divergent; like geography it is a loose federation of interests without a methodology or a philosophy in common. The field is, fortunately, quite 'rural'; there are plenty of different topics to discuss and no great competition to come up with the answer before other workers. Areas of it are reasonably 'hard': we know quite a lot more about text structure and register than we used to, and applications like lexicography for example, seem to make definite progress. There would be more cumulation if LSP scholars were better at reading one another's work and adopting standard analytic categories

Furthermore, if one is working in the 'hard' quantitative end of LSP one should be consistent. The other two articles from Gunnarson's project, reporting actual numbers, are interesting but share with several other quantitative papers in the volume a surprising weakness: by not applying a standard of statistical significance they throw away half the content of their data. Use of such a measure would enable researchers to say that while features $\mathrm{X}$ and $\mathrm{Y}$ are different between their two samples, $\mathrm{Z}$ is essentially the same. It is a pity to exclude the possibility of saying that $\mathrm{Z}$, contrary to a natural assumption, is not 
indexical of anything; this seems as important as finding that $\mathrm{X}$ and $\mathrm{Y}$ are indeed indexical.

LSP's commitment to the useful arts is underlined by another historical paper, remote though such studies may seem from the daily lives of practitioners dragging unwilling bilingual secretaries and civil engineers through the rudiments of business or technical language. McKinnon looks at Sprat's early eighteenth-century History of the Royal Society and shows how the Society's programme was already characterised by LSP values: utilitarianism, a demand for terminological precision, and the marriage of science and commerce. Gläser looks at the application of this programme, comparing medical articles from the first Encyclopaedia Britannica with their counterparts in the most recent edition She is able to show linguistic indices of increased impersonality, of professionalisation, and of paradigm shifts. It is particularly interesting that she finds eighteenth-century medicine using forrns of appeal to other specialists' work which emphasise the authority of known individuals rather than the value of documented results despite the Royal Society's rejection of this kind of authority.

Many papers deal with LSP as a 'liberal' rather than a 'useful' art and focus on its implications for the philosophy of science, sociology, etc. rather than any possible application. The most ambitious is de Beaugrande's plenary, which demonstrates a critical analysis of the $\mathrm{D}$ (discourse)SP of Piaget. The analysis is 'post-classical' in the sense that it aims at avoiding reductionism (a simple model with a complex relation to its domain) and producing a complex model simply related to its domain. The result is a methodology so full of metaphor and complexity that it is difficult to follow without oneself reducing it. However de Beaugrande produces many interesting observations including the notions of controlling concepts, manifested throughout the vocabulary of the text and thus driving it forward to its conclusion, and that of 'putting pressure on the text' to draw out the ideology. This either results in increasing coherence (perceiving an overall control system with an integrated set of central terms, so that each claim matches the agenda even if it seemed odd to start with) or decreasing it - revealing that a perfectly consistent claim is rejected just because made by a rival, etc.

The highly metaphorical nature of de Beaugrande's language raises the theme of metaphor in specialist language, perhaps quite fashionable in the wake of the cognitive linguists, which appears in papers in several sections. (The contrast between the Royal Society's Enlightenment suspicion of figurative language and this 'post-classical' trend is significant). Lorenz and Lorenz look at the way metaphors were used to humanise computers, and how the computer has become a metaphor for humans in its turn; Corbisier evaluates the communicative effectiveness of explanatory analogies in computer 
manuals (and finds it very variable); Kocourek similarly develops criteria for evaluating the appropriacy of metaphors which become terminologised, an important issue in view of the power of the metaphor to shape thinking on the topic though the resulting list is difficult to interpret; and Stålhammar draws conclusions about ideology from personifications of the market in Swedish and English newspapers. All these articles, like others in the volumes, would have benefited from a little more of the Royal Society spirit of 'founding' knowledge not only on 'thoughts and words' but also on 'works', in this case empirical analysis of the reception of metaphors by readers.

As Stålhammer shows, the form of discourse is related to the underlying ideology. Several papers look at the way journalistic writing reveals its ideological bias. One of the most interesting is Ledin's on changes in the types of polyphony in the turn-of-the-century socialist press in Sweden. His genre model allows a focus on 'voice' which links with studies in other fields. However the most fruitful approach is Benke's, studying the nature of the news agency reports which underlie newspaper articles and the way that their biases are amplified by the editing process. The process by which ideologicallyloaded texts are created is of more interest really than the fact that texts are ideologically-loaded, particularly if one wants to understand how bias becomes naturalised for the journalists. On the other hand, calling newspaper language LSP seems to stretch the category almost to breaking point: "specialist communication' addressed to a mass public seems a contradiction.

Among the 'hardest' work in the conference in the sense of being part of a cumulation of knowledge, there is a large group of papers which, without ignoring ideological implications, report characteristics of important types of texts and make the knowledge accessible for application in teaching or translation. Of those dealing with academic, often research, writing, Hunston's is one of the most interesting. It identifies various formal features related to modality or truth-value, suggests characteristic speech-act roles for them, and shows how, because of the uses of these roles in advancing claims, the forms tend to appear in particular positions in research articles. The result enables the reader of a research report to evoke a 'mesh of knowledge' from familiar forms whose roles are necessarily related to the formation of scientific understanding. The paper is qualitative so we do not know what proportion of actual papers conform to the pattern in her extended example from a Discussion section, but its approach is illuminating and the findings immediately applicable. Banks' work on hedging, Schramm's on tense and aspect, and Lindberg's on the conventions of conclusions in finance, management and marketing research are of similar quality and similarly cumulative. Kourilova's article on modality in biomedical research reporting is less well integrated with the literature but makes nice points with wide implications; for example text- 
books make causal connections explicit while research articles leave them to be inferred from disciplinary knowledge.

Examining legal German, Engberg applies this type of analysis to the complex relations of levels which characterise text. Again, linguistic forms do not signal speech acts, and these are not in one-to-one relation with text parts, but the necessary repetitiveness of specialised activities results in regular associations. Thus Engberg finds zur Uberzeugung der Kammer/des Gerichts in 11 of his 30 judgements, and this is exactly the kind of frequency one expects where writers are not bound by formulae but influenced by previous texts in the same tradition.

A recent special issue of English for Special Purposes (St. John 1996) refers to the explosion of interest in business LSP in the 1990s, but, though teaching is well represented, as usual, (Yli-Jokipii 1996) there is limited evidence of research in this conference. Fløttum et al give a cross-linguistic comparison of the style and genre conventions of 'Chairman's statements' in public company reports (five each in English, French, and German); without giving figures, they find, for example, much more paratext in the German examples than in the French, and more direct openings and less appeal to the reader in French than in German and English. Three papers deal with aspects of oral negotiation in a second language on the basis of transcripts. Neumann finds a number of differences between Germans and Norwegians in the use of German metadiscoursal phrases. Öberg looks at miscommunication in English-language negotiations among a variety of nationalities and finds that semantic and discourselevel breakdown is recognised and repaired, while miscommunication on the level of culture and presupposition requires thematisation and discussion if it is to be overcome, and this is quite often not achieved. The third paper, by Wilkinson et al discusses the optimisation of redundancy in intercultural discussion (ways, that is, to minimise both miscommunication and longwindedness) and sets out a programme of research to identify and teach important characteristics.

Empirical reports of the development of new LSPs or new aspects of LSP are an important function of a collection like this, alerting practitioners to new training or translation needs. The most revealing here is Johnson's straightforward account of the problems arising from the multiple ambiguity and richly figurative language of radio communication among Kent policemen and the consequent development of a reduced subset of English - Policespeak - for use in the Channel Tunnel. In an inspired piece of observation, Naylor describes the way that teleconferencing in English demands that a speaker in one town 'point' to a picture in another, and so favours those who are able to develop a new type of 'deictic' emphasis in their speech. Tveit describes the language of subtitling and the particular translation/adaptation processes in- 
volved. In a similar spirit, Jarvi, Draskau, Picht and Budin provide varying typologies and terms for including nonverbal representations in the scope of LSP, given their central role in technical communication. Draskau's point that we should therefore speak of 'specialist communication' rather than LSP is well taken.

The terminology papers make a bridge between 'pure' research and the applications. Picht's exciting overview of terminology in 1993 sees the field as divided into 'pure' theory (with implications for the philosophy of science, and semiotics) and applications, increasingly involving electronic media: he lists translation and technical writing, terminology planning, constantly updated term banks for terminography, knowledge engineering, information science and information management, etc. Callewaert and Markey exemplify one of these applications with their report on a computer-based multilingual thesaurus of business communication. Good introductions are provided by Grinev's plenary on the basic principles and classification of (Russian) terminology, and Rogers and Ahmad's paper, which presents terminological principles for translators (and then their programme for computer-assisted bilingual terminography). Jemudd's plenary sets these in a context of application by showing that one external factor which interacts with terminology in application is politics: francization in Quebec; the conflict between spontaneous 'uneducated' terminologies (of motor maintenance in Mexico, for example) and prescriptive 'academic' ones; conflicts between international terminological agreements and the perceived political significance of their terms. A variety of other factors emerge; Jernudd cites examples from the practice of the Swedish Center for Technical Terminology, many of them legal: what counts as a facade? what does diagonal mean?

Other papers give information about the structure of technical language which is useful in many applications: Nuopponnen classifies types of causality in 'multidisciplinary' fields; Puuronen develops a framework for discussing technical verbs (rather than nouns); and, most illuminatingly, van der Staak analyses the speech act EXPLAIN in technical writing. Van der Staak identifies the possible types of explanation in terms of method/strategy, (with a list of illocutionary indicators), type (representative vs. declarative), degree of explicitness, perspective (word-focus, referent-focus), and order of ideas.

Two keynote talks dealt with applications to LSP teaching. Huckin called for a 'narrow-angle' approach finely tuned to particular situations, seeing this as part of a development in a variety of fields towards particularity and embedding of description in the context. This can be related to the anti-reductionism of de Beaugrande's presentation; it also suggests that these and other text-analytic insights will be useful to the dedicated ESP teacher rather as models of analysis and hints as to what will be found than as direct input to teaching 
materials. Huckin regrets that ESP teaching has not developed a theoretical base or its own set of methodological principles, but this is a reflection of an odd stance that LSP teaching is somehow a different field from languageteaching; in fact the rich toolbox of communicative techniques has by no means been fully exploited, and pedagogy is to do with psychology rather than technical-communication studies. Most LSP researchers are also teachers, translators, or lexicographers, but in this second role they have to draw on two disciplines: the LSP branches of linguistics, and the relevant branches of pedagogy, translation studies, etc. Failure in the parent discipline leads to problems in the application; one can relate Puuronen's observation that terminologists have ignored verbs to Stephenson's report of her students' ignorance of the verbs that go with the nouns they have been carefully taught.

Salager-Meyer's plenary on LSP teaching in higher education in the third world raises the political issues around LSP's concentration on powerful postimperial languages, which are otherwise to little discussed. How, she asks, can LSP in these circumstances help overcome domination? The practice of teaching large groups of first-year Spanish-speaking students English (usually) for academic purposes before they need it is politically questionable and usually highly inefficient. It would be better to concentrate on graduates and staff with definable needs and mature attitudes. Actually the whole issue of specialisation is to do with exclusion (a Fach is according to Kalverkämper surrounded by a fence of Fachwerk!) and issues of popularisation and inclusion should be on the LSP agenda.

McGinity et al on Spain, Oladejo on New Guinea and Love and Stephenson on Zimbabwe similarly reflect the depressing task of the teacher of EAP to demotivated students whether English is the medium of instruction (and hence students believe they know it), or English is a compulsory extra (and students don't believe they need it). However Love's incisive analysis of geomorphological discourse and corresponding development of classroom activities to guide interactive reading shows that Huckin's particularity is indeed a way forward. This paper also represents the ideal movement of LSP: from the sociolinguistic environment, to text analysis, to classroom procedures. Huckin's other theme of methodological innovation is brilliantly illustrated in the poor conditions of first-year EVP teaching for classes of 150 by RamonSales. She had her classes of Spanish students of medicine, librarianship, and business write weekly letters to one another. Every medic and librarian enquired about materials from a business student, got a reply, ordered, got a delivery note, complained, and got an apology. The students knew they would have to do this in real life (and, as often happens, were anyway more interested in each others' specialities than their own). If students have real current needs, that is, one must respond to them precisely; if their needs are in an indistinct 
future, face validity and communicative liveliness are more important.

The opposite pole from large-class, low-tech, low-motivation LSP is represented by several reports of technical innovations, most of them relevant to any type of language teaching. Sanne provides a useful description of software for enabling self-access use of videodisks. Baten describes and evaluates two vocabulary programmes. His conclusion that encouraging students to use the potential of the systems requires immense clarity in the hypertext environment, careful training, staging of program complexity, and frequent monitoring needs to be borne in mind by all designers; clever program design like that of Buhl and Johnsen's 'LSP Student's Workbench' is unfortunately only the first stage.

As with the text-descriptions, there are so many pedagogic articles giving useful information that they cannot all be discussed. Lillie deals with the problems of introducing case studies and semi-independent study in a course in language for business. Despite the independent-study ideology of the teachers, what the students really perceived as useful was unprepared interpreting practice. Lillie envisages a cautious route forward, educating the learners in the ideology of independence without ignoring their perceptions. SolerSeaone provides a model for the rarely-taught topic of language for mathematics. Jonathan Shaw's approach to teaching the research literature review is another fine example of descriptive research incorporated into innovative pedagogy. Markey's questionnaire for the evaluation of business letters offers a framework for escaping from marking grammar errors, though her examples show how much subjectivity must enter into judgements of more important but less concrete mistakes in 'appropriacy', etc. There is an urgent need for detailed corpus-based descriptions of what does occur, so that questionnaires like this can be based on saying '99\% of French business letters don't do this' rather than 'I feel this is wrong'. Finally Sanctobin and. Slootmaekers' paper on learners' written and oral description of graphs, the only study of LSP acquisition in the book, reports that training in specific language items continues to confer an advantage even as task difficulty increases; it is not merely decoration to be abandoned as things get harder. LSP acquisition is a field worth developing.

Bilingual lexicography is represented by a few interesting papers. Duvå, Laursen, and Maidahl show that dictionaries on an alphabetical (semasiological) basis leave out quite a lot of important words in fields like accounting, and anyway fail to account for polysemy and homonymy, especially where it is the same in both languages (the reader cannot know whether it is or not). So the most useful form of LSP bilingual dictionary, they argue, would be onomasiological/ terminological, organised by area, with related terms together, and an alphabetical index. This would guarantee completeness and would give 
translators a chance to see what a particular term was doing i.e. what it was in contrast with. Agirre et al describe a computer-based intelligent dictionary help system (French-Basque), one not based on word-for word equivalence but on the system's 'knowledge' of the semantic structure of the two languages.

Like Salager-Meyer's plenary, the third lexicographical paper brings us up against the problems concealed by LSP's focus on cold rich countries and big languages. Schaarschmidt compares German-Sorbian dictionaries from 1920 and 1989. Sorbian is in the position of Scots Gaelic or Vlach; all speakers are bilingual and the language is dying. The only LSP issue raised is how far one should coin minority-language words for things like 'cold rolling mill' that the speakers will undoubtedly use the majority term for; the wider issue is how exclusively resources in teaching and research are directed to the economically or at least politically important languages and not, for example, to the preservation of diversity.

A final area of application is translation, which is rather under-represented in this collection. Anyone using the section to get an overview of activity in translation studies would do well to start with Kvam's clear exposition of the issues surrounding equivalence, adequacy, relevance, etc. Accepting the primacy of preserving the skopos, he uses the term Loyalität for the 'primary text relation' between source and target text, involving first of all Kompatibilität to ensure that the target text is indeed a translation, and then some degree of Korrespondenz, as required by the commission. The other papers in this section range from very 'soft' to very 'hard' - from atheoretical examples of problems in particular cases to technical discussion of machine translation. In the middle is Halverson's qualitative analysis of translations of some types of Norwegian clause into English, using a panel of American students to judge the acceptability of the results. She found very different 'styles' with respect to word-level equivalence among translators, and some differences among source structures in their susceptibility to rank-shifted translation. The 'hardest' papers deal with machine translation: O'Brien lists the differences between two sorts of text as a prerequisite for MT. Thunes provides an introductory comparison of two machine translation strategies - transfer which goes directly from L1 to L2, and interlingua, which makes use of a language-neutral interlingual code - and describes systems being developed in Norway.

On first reading these proceedings, one has a sense of varied quality, with rather few highlights, and of a conference full of people with varied interests and concerns talking past one another. But if one views the field as the editors do, with a central core of register/text/discourse analysis reaching out to philosophy and critical theory in its implications and supplying a variety of practical fields with information in its applications, the papers begin to illuminate one another, so that developments in one area suggests possibilities in 
another. The terminological papers suggest the need for more research on the structure of learner's LSP vocabularies; the teaching ones raise questions about the sociolinguistics (the power relations) in LSP, and the whole volume shows how much the applied disciplines need the 'pure' researchers to carry out rigorous analysis in comparable terms of a wider variety of specialist communication in a wider variety of languages.

\section{References}

Becher, T. (1989): Academic Tribes and Territories. Milton Keynes: Open University Press.

Kalverkamper (1992): Die kulturanthropologische Dimension von 'Fachlichkeit' im Handeln und Sprechen. Fachsprache und Terminologie in Geschichte und Gegenwart. J. Albrect and R. Baum. Tuebingen: Gunter Narr: 3-57.

St.John (1996): Business is booming: business English in the 1990s. In: English for Specific Purposes 15(1): 3-18.

Yli-Jokipii, H. ( 1996): An approach to contrasting languages and cultures in the corporate context: Finnish, British, and American business letters and telefax messages. In: Multilingua 15(3): 305-327. 